\title{
Whole-Genome Sequence Analysis of Human Papillomavirus Type 18 from Infected Thai Women
}

\author{
Woradee Lurchachaiwong ${ }^{a, d}$ Pairoj Junyangdikul ${ }^{\mathrm{e}}$ Wichai Termrungruanglert $^{\mathrm{b}}$ \\ Sunchai Payungporn ${ }^{\mathrm{a}}$ Pichet Sampatanukul $^{\mathrm{c}}$ Damrong Tresukosol ${ }^{\mathrm{b}}$ \\ Somchai Niruthisard ${ }^{b}$ Prasert Trivijitsilp ${ }^{b} \quad$ Anant Karalak $^{f}$ \\ Sukumarn Swangvaree ${ }^{g}$ Yong Poovorawan ${ }^{a}$ \\ ${ }^{a}$ Center of Excellence in Clinical Virology, and Departments of ${ }^{b}$ Obstetrics and Gynecology and ${ }^{c}$ Pathology, \\ Faculty of Medicine, and IInterdepartment of Biomedical Sciences, Graduate School, Chulalongkorn University, \\ e Department of Pathology, Samitivej Srinakharin Hospital, and Departments of ${ }^{f}$ Gynecologic Oncology and \\ gPathology, National Cancer Institute, Bangkok, Thailand
}

\section{Key Words}

Human papillomavirus type $18 \cdot$ Whole genome, characterization

\begin{abstract}
Objective: The aim of this study was to attain molecular knowledge of human papillomavirus type 18 (HPV18) by sequencing the whole genome of HPV18 isolated from Thai women at various clinical stages of disease progression. Method: Our group analyzed 9 samples of whole-genome HPV18 in infected women ranging from normal to cervical cancer by PCR, a sequencing method and bioinformatics programs. Results: Phylogenetic analysis based on the whole genome showed that HPV18 samples were more closely related to the European and Asian-American type than the African type. The vaccine strain's L1 nucleotide (US patent 5820870) showed a close relationship to the African type. However, our data cannot indicate the correlation between cytological data and nucleotide or amino acid variation. Conclusion: Our group cannot draw any inference be-
\end{abstract}

tween the clinical stage of disease progression and amino acid alterations as there were only 1 or 2 samples available for each clinical trial. However, we hope that these new data on the HPV genome, which are representative of the entire genome of HPV in Southeast Asia, can serve as basis data for future research on the pathogenesis of cervical cancer. Additionally, the second-generation HPV18 vaccines should be tested on both HPV18-L1 and HPV18-L2 for increasing potential protection.

Copyright $\odot 2010$ S. Karger AG, Basel

Human papillomavirus type 18 (HPV18) is the second most prevalent genotype found in cervical cancer [1]. HPV18 has numerous variants which have been identified in different geographical locations [1-3]. Since the HPV18 genome displays numerous variations, HPV18 can be classified as a variant of three distinct groups: European, Asian-American and African [4]. HPV18 variants are associated with progression of cervical neoplasia, and the diversity of the HPV18 oncogenic potential de-

\section{KARGER}

Fax +41613061234 E-Mail karger@karger.ch www.karger.com
(C) 2010 S. Karger AG, Basel

$0300-5526 / 10 / 0533-0161 \$ 26.00 / 0$

Accessible online at:

www.karger.com/int
Prof. Yong Poovorawan

Center of Excellence in Clinical Virology, Department of Pediatrics

Faculty of Medicine, Chulalongkorn University

Bangkok 10330 (Thailand)

Tel. +66 2256 4909, Fax +66 2256 4929, E-Mail Yong.P@ @ chula.ac.th 
Table 1. Conserved primers for whole-genome amplification and sequencing of HPV18

\begin{tabular}{|c|c|c|c|c|}
\hline Set & Primer name & Sequence (from $5^{\prime}$ to $3^{\prime}$ ) & Position & Product, bp \\
\hline \multirow[t]{2}{*}{$\mathrm{A}$} & F15E6_HPV18 & CAATTgTAgTATATAAAAAAgggAgT & 15 & 886 \\
\hline & R901E6_HPV18 & ggATgCACACCACggACACAC & 901 & \\
\hline \multirow[t]{2}{*}{$\mathrm{B}$} & F828E7_HPV18 & CAgACgACCTTCgAgCATTCCA & 828 & 803 \\
\hline & R1683E1_HPV18 & TTTACAgTCTAgACATTgAATATgg & 1631 & \\
\hline \multirow[t]{2}{*}{$\mathrm{C}$} & F1612E1_HPV18 & AAAgTgATAAAACCACgTgTACAg & 1612 & 896 \\
\hline & R2508E1_HPV18 & gTTgCATCATCTAACATggCCAC & 2508 & \\
\hline \multirow[t]{2}{*}{$\mathrm{D}$} & F2458E1_HPV18 & TTggTTggAACCgTTAACAgATAC & 2458 & 935 \\
\hline & R3393E2_HPV18 & AATgTACTTCCCACgTACCTgTg & 3393 & \\
\hline \multirow[t]{2}{*}{$\mathrm{E}$} & F3160E2_HPV18 & AAAggTggCCAAACAgTACAAgTA & 3160 & 969 \\
\hline & R4129E4_HPV18 & TgCAATAgTAACATgggCAAT & 4129 & \\
\hline \multirow[t]{2}{*}{$\mathrm{F}$} & F4016E4_HPV18 & TATgTgTgCgTATgCATgggTAT & 4016 & 977 \\
\hline & R4993L2_HPV18 & TgTAATTAAAgAggATggACgTgT & 4993 & \\
\hline \multirow[t]{2}{*}{ G } & F4871L2_HPV18 & CATTAgTAgTACCCCATTgCCTA & 4870 & 954 \\
\hline & R5824L2_HPV18 & CTATATTggTATgCAgAAACCTTAg & 5824 & \\
\hline \multirow[t]{2}{*}{$\mathrm{H}$} & F5757L2_HPV18 & AgATTATTAACTgTTggTAATCCATA & 5757 & 911 \\
\hline & R6668L1_HPV18 & TACAggAgACTgTgTAgAAgCAC & 6668 & \\
\hline \multirow[t]{2}{*}{ I } & F6538L1_HPV18 & ggCTCTATTgTTACCTCTgACTC & 6538 & 894 \\
\hline & R7432L1_HPV18 & ggATAAAATggATgCTgTAAggTg & 7432 & \\
\hline \multirow[t]{2}{*}{$\mathrm{J}$} & F7296L1_HPV18 & gTTCTgTgTgTTATgTggTTgCg & 7296 & 734 \\
\hline & R173E6_HPV18 & AgTgTTCAgTTCCgTgCACAgAT & 173 & \\
\hline
\end{tabular}

Position is based on the nucleotide sequence of HPV18 (X05015).

pends on geographical area and ethnicity of the population studied [5, 6]. HPV18 can be found in both squamous cell carcinoma and adenocarcinoma. However, HPV18 is more frequent in cervical adenocarcinoma than any other viral type, while HPV16 is more frequent in squamous cell carcinoma $[7,8]$. HPV18 variants display different potentials of cancer progression. A previous study has revealed that infection by European variants tends to persist [9], whereas Asian-American variants induced tumors in vivo [5]. Moreover, HPV18 variants play different roles in the pathogenesis of cervical cancer [10]. Some previous projects have studied HPV16 at different stages of cervical cancer by examining the distribution of the long control region, E7, E6, L1, L2 and E2 polymorphisms. Thus, we have decided to examine the nucleotide and amino acid variations of HPV18 in critical regions as described in previous studies $[1,11]$. The present research has been aimed at characterizing the whole genome (long control region, LCR; early gene and late gene) variability of HPV18 in Thai women at different clinical stages of cervical cancer based on cytological findings by focusing on the critical point. This project presents whole-genome results representative of Southeast Asia in comparison with HPV18 variants from other countries. Finally, it will provide useful information for identifying the nucleo- tides associated with viral function, viral persistence and pathogenicity.

The study protocols were approved by the Ethics Committee of the hospital and Faculty of Medicine, Chulalongkorn University. All specimens were collected for cytology by liquid-based cytology (Thinprep ${ }^{\circledR}$; Hologic, UK) and tested for HPV DNA by using Hybrid capture II (Digene). Subsequent to BLAST analysis of the whole genome amplified by polymerase chain reaction (PCR), the respective genotypes were determined by direct sequencing. All HPV samples were stored at $-70^{\circ}$ until used. From 515 samples, 16 were classified as CA group, with HPV18 present in 3 samples $(3 / 16,18.75 \%)$, 98 samples were classified as HSIL group, with HPV18 found in 3 samples (3/98, 3.06\%), 136 samples were classified as LSIL group, with HPV18 detected in 2 samples (2/136, 1.47\%), 186 samples were classified as normal group, with HPV18 present in 3 samples $(3 / 186,1.61 \%)$. Thus, we randomly selected HPV18-positive Thai women representing patients with different cytological data. DNA was obtained by organic extraction (phenol-chloroform) of the samples as described previously [12]. PCR was performed to amplify the HPV genome. The reaction mixture comprised $2 \mu \mathrm{l}$ DNA, $0.5 \mu \mathrm{M}$ of each primer shown in table $1,10 \mu \mathrm{l}$ of $2.5 \times$ Eppendorf master mix (Eppendorf, Hamburg, 
Germany) and nuclease-free water to a final volume of $25 \mu$ l. The amplification reaction was performed in a thermal cycler (Eppendorf) under the following conditions: initial denaturation at $94^{\circ}$ for $3 \mathrm{~min}$, followed by 40 amplification cycles consisting of denaturation at $94^{\circ}$ for $30 \mathrm{~s}$, primer annealing at $55^{\circ}$ for $45 \mathrm{~s}$ and extension at $72^{\circ}$ for $1.30 \mathrm{~min}$, and concluded by a final extension at $72^{\circ}$ for 7 min. HPV primer positions and PCR products are depicted in table 1 . The housekeeping gene $\beta$-globin was selected to serve as an internal control for DNA extraction, using conventional PCR as a detection method. Primer sequences for the $\beta$-globin gene have been previously described [13].

The PCR products were obtained as described above and used for directed sequencing. Determination of the nucleotide sequences was performed in duplicate and analyzed in both directions using forward and reverse primers to ensure that variations of nucleotide sequences were not due to sequencing errors. When a difference was observed, triplicate sequences were determined in order to confirm the consistency of the sequencing result. Nucleotide sequences were analyzed and assembled using the Lasergene 6 Package ${ }^{\circledR}$ (DNAstar Inc., Madison, Wisc., USA) and BLAST analysis tool (http://www.ncbi.nlm. gov/BLAST). Complete genome sequences were prepared and aligned using Clustal W applied by the Bioedit program (version 7.0.4.1). Phylogenetic trees were constructed by neighbor-joining analysis with the Tamura-Nei model executed by the Mega $3^{\circ}$ program [14]. The rate between nonsynonymous ( $\mathrm{dN}$ ) and synonymous (dS) substitutions was measured by the single likelihood ancestor counting method in Datamonkey elsewhere [15]. Rates of $\mathrm{dN} / \mathrm{dS}$ refer to selective pressure acting on a protein-coding gene. A $\mathrm{dN} / \mathrm{dS}$ ratio $>1$ indicates that this gene is evolving under positive selection. All nucleotide sequences of HPV18 obtained in the course of this project were submitted to the Genbank database under designated accession No. GQ180784-92.

Nine HPV18-positive samples (CU8-CU16) displayed different cytology results such as normal (CU8-CU9), LSIL (CU10-CU11), HSIL (CU12-CU13), squamous cell carcinoma (CU14-CU15) and adenocarcinoma (CU16). HSIL and cervical cancer (CU15-CU16) samples have a histological outcome to confirm the cytological result whereas normal and LSIL samples lacked histological outcome. This study was performed on women between 19 and 70 years of age. All 9 samples had shown positive results on application of a commercially available test kit (Hybrid capture II) except for CU14 and CU16 which had yielded an abnormal Pap smear detected by a cytopathol- ogist. Whole-genome sequences of HPV18 found in Thai samples (accession No. GQ180784-92) were aligned with X05015 [16], NC001357, Y262282, EF202152-EF202155 (African type), EF202147-EF202151 (European type) and EF202143-EF202146 (Asian-American type). Analysis of HPV nucleotide sequences showed 97.9-99.9\% similarity among the Thai and all reference sequences (data not shown). Phylogenetic analysis revealed that HPV18 in Thailand was closely related with European and AsianAmerican types (fig. 1).

The complete genome of CU11 comprised 7,844 bp, whereas other samples consisted of 7,857 bp indicating variable genome lengths of HPV18 isolated from Thai women. We observed deletion in the LCR (7245-7256) and E4 gene (position 3630-3635) of CU11 samples (data not shown). These deletions occurred in the African type, and phylogenetic tree analysis showed that CU11 was closely related with the African type whereas other samples were related with the European and Asian-American types (fig. 1). In contrast to existing data on HPV16 variants, HPV16 in Thailand was more closely related to the European than the Asian-American and the African types [17]. Thus, our study showed that HPV18 variants in Thailand related two distinct groups of HPV18 variants (European and Asian-American types). Nevertheless, due to the small sample size, this finding should be verified by larger studies.

Based on nucleotide variation, we focused on the LCR segment at positions 41, 104 and 7,726. A previous study had reported that nucleotide variations in this segment should affect transcription factor binding sites such as Sp1 (selective promoter factor 1), YY1 (yin-yang factor 1) and Oct1 (octamer binding factor), respectively [1]. Based on our results, nucleotide variations at these critical positions occurred with 2 samples (CU10 and CU11). Nucleotide variation of CU10 changed both positions 41 (A to $G$ ) and 104 ( $\mathrm{T}$ to $\mathrm{C}$ ), whereas the nucleotide sequence of CU11 changed at positions 104 ( $\mathrm{T}$ to $\mathrm{C}$ ) and 7726 (C to $\mathrm{T})$. Variation of nucleotides 41 and 104 was associated with a higher activity of the E6/E7 promoter by modulating Sp1 and YY1 activities [11]. A previous report indicated that nucleotide alterations at position 104 were less likely to promote tumor recurrence than virus with the reference sequence [11] and variation was usually found in the European type. Based on the pattern of nucleotide variation, all samples displayed more similarity to the European (especially CU10) and Asian-American type than the African type. We could detect these changes in CU10 and CU11 that indicated LSIL cytological data. 
Fig. 1. Phylogenetic tree based on wholegenome analyses of 9 samples and reference sequences from the Genbank database. Phylogenetic tree construction by neighbor-joining analysis with the Kimura model implemented in the $\mathrm{Mega}^{\odot}$ program (version 3.1).

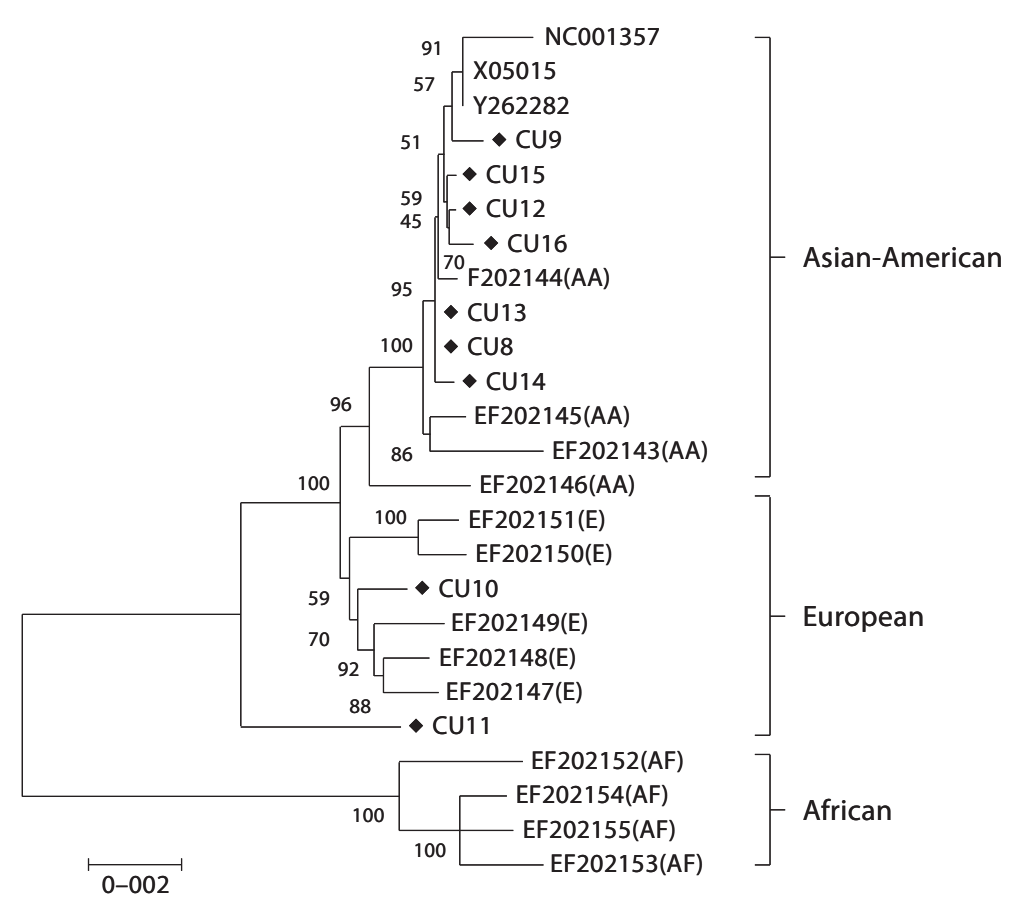

cant for all coding regions. Thus, our data did not perform and analyze amino acid variation on the E2 gene. However, E2 variation could affect other biological functions such as viral replication or interaction with other cellular proteins [19].

Alignment of the vaccine strain's (US patent 5820870) polypeptide with all our samples has shown that the L1 vaccine strain is closely related to the African type, whereas most of our samples' sequences are related to the Asian-American and European types. Some previous studies revealed that oncogenic potential and HPV18 variants demonstrated that the African type is usually found in patients with less abnormal pathology [21]. Although bivalent and quadrivalent vaccine cross-reactivities have been reported, long-term stimulation of immune response remains to be established in direct comparison [22]. A report revealed that protective and broadly cross-neutralization should be emphasized on L2 [23]. Thus, a second-generation HPV18 vaccine should be tested on both HPV18-L1 and HPV18-L2 for increasing potential protection. Due to our small sample size, we could not observe any significant correlation between HPV18 variants detected in this study and the results 
from cervical cytology. In addition, we did not see any difference in the distribution of HPV18 variants when comparing squamous cell carcinoma with adenocarcinomas. Our data support the proposed association of nonEuropean variants (especially the Asian-American variant) with adenocarcinoma as suggested elsewhere [24]. However, our project was limited by the small sample size. It had been intended as a preliminary study aimed at HPV18 genome characterization in Thailand which would represent HPV18 in Southeast Asia.

In conclusion, we found numerous nucleotide and amino acid variations in the genome of HPV18 isolated from infected Thai women. Whole-genome analysis of these samples showed HPV18 from Thailand to be more closely related to the European and the Asian-American than African type. This study revealed that our group cannot draw any inference between the clinical stage of disease progression and amino acid alterations as there were only 1 or 2 samples available for each clinical trial. However, we hope that these new data on the HPV18 genome which are representative of the entire genome of HPV18 in Southeast Asia can serve as basic data for scientific research on the pathogenesis of cervical cancer.

\section{Acknowledgements}

We would like to express our gratitude to the Thailand Research Fund (Royal Golden Jubilee PhD Program), the Commission on Higher Education, Ministry of Education and the Center of Excellence in Clinical Virology, Chulalongkorn University and Hospital, for their generous support and the staff of the Department of Pathology, Samitivej Srinakharin Hospital, Thailand, for providing the samples. Finally, we also would like to thank Ms. Petra Hirsch for reviewing the manuscript.

\section{References}

1 Cerqueira DM, Raiol T, Véras NM, von Gal Milanezi N, Amaral FA, de Macedo Brígido M, Martin CR: New variants of human papillomavirus type 18 identified in Central Brazil. Virus Genes 2008;3:282-287.

2 Ho H, Chan SY, Burk RD, Das BC, Fuginaga K, Icenogle JP, Kahn T, Kiviat N, Lancaster W, Mavromara-Nazos P, Labropoulou V, Mitrani-Rosenbaum S, Norrild B, Pillai MR, Stoerker J, Syrjaenen K, Syrjaenen S, Tay SK, Villa LL, Wheeler CM, Williamson AL, Bernard HU: The genetic drift of human papillomavirus type 16 is a means of reconstructing prehistoric viral spread and the movement of ancient human populations. J Virol 1993;67:6413-6423.

- 3 Yamada T, Manos MM, Peto J, Greer CE, Muñoz N, Bosch FX: Human papillomavirus types 16 sequence variation in cervical cancers: a worldwide perspective. J Virol 1997; 71:2463-2472.

4 Ong CK, Chan SY, Campo MS, Fujinaga K, Mavromara-Nazos P, Labropoulou V, Pfister H, Tay SK, Meulen JT, Villa LL: Evolution of human papillomavirus type 18: an ancient phylogenetic root in Africa and intratype diversity reflect coevolution with human ethnic groups. J Virol 1993;67:6424-6431.

5 Villa LL, Sichero L, Rahal P, Caballer O, Ferenczy A, Rohan T: Molecular variants of human papillomavirus types 16 and 18 preferentially associated with cervical neoplasia. J Gen Virol 2000;81:2959-2968.

-6 Sichero L, Ferreira S, Trottier H, DuarteFranco E, Ferenczy A, Franco EL: High grade cervical lesions are caused preferentially by non-European variants of HPVs 16 and 18. Int J Cancer 2007;120:1763-1768.
7 Bulk S, Berkhof J, Bulkmans NW, Zielinski GD, Rozendaal L, Van Kemenade FJ, Snidjers PJ, Meijer CJ: Preferential risk of HPV16 for squamous cell carcinoma and of HPV18 for adenocarcinoma of the cervix compared to woman with normal cytology in the Netherlands. Br J Cancer 2006;94:171-175.

8 Burk RD, Terai M, Gravitt PE, Briton LA, Kurman RJ, Barnes WA, Greenberg MD, Hadjimichael OC, Fu L: Distribution of human papillomavirus types 16 and 18 variants in squamous cell carcinomas and adenocarcinomas of the cervix. Cancer Res 2003;63: $7215-7220$.

-9 de la Cruz-Hernández E, García-Carrancá A, Mohar-Betancourt A, Dueñas-González A, Contreras-Paredes A, Pérez-Cardenas E, Herrera-Goepfert R, Lizano-Soberón M: Differential splicing of E6 within human papillomavirus type 18 variants and functional consequences. J Gen Virol 2005;86: 2459-2468.

10 de Boer MA, Peters LA, Aziz MF, Siregar B, Cornain S, Vrede MA, Jordanova ES, Fleuren GJ: Human papillomavirus types 18 variants: histopathology and E6/E7 polymorphisms in three countries. Int J Cancer 2005; 114:422-425 (erratum published in Int J Cancer 2005;114:1016).

11 Rose BR, Thompson CH, Zhang J, Stoeter M, Stephen A, Pfister H, Tattersall MH, Cossart YE: Sequence variation in the upstream regulatory region of HPV 18 isolates from cervical cancers. Gynecol Oncol 1997;66:282-289.
12 Broccolo F, Drago F, Careddu AM, Foglieni C, Turbino L, Cocuzza CE, Gelmetti C, Lusso P, Rebora AE, Malnati MS: Additional evidence that pityriasis rosea is associated with reactivation of human herpesvirus- 6 and -7 . J Invest Dermatol 2005;124:1234-1240.

13 Shadrina MI, Semenova EV, Slominsky PA, Bagyeva GH, Illarioshkin SN, Ivanova-Smolenskaia II, Limborska SA: Effective quantitative real-time polymerase chain reaction analysis of the parkin gene (PARK2) exon 1-12 dosage. BMC Med Genet 2007;8:6.

14 Kumar S, Tamura K, Nei M: Mega3: integrated software for molecular evolutionary genetics analysis and sequence alignment. Brief Bioinform 2004;5:150-163.

15 Pond SL, Frost SD: Datamonkey: rapid detection of selective pressure on individual sites of codon alignments. Bioinformatics 2005;21:2531-2533.

16 Myers G, Delius H, Icenogle J, Bernard HU, Favre M, van Ranst M, Wheeler CM: Human papillomaviruses 1997: a complication and analysis of nucleic acid and amino acid sequences. Los Alamos, Theoretical Biology and Biophysics, Los Alamos National Laboratory, 1997.

17 Lurchachaiwong W, Junyangdikul P, Payungporn S, Chansaenroj J, Sampathanukul P, TresukosolD, TermrungruanglertW, Theamboonlers A, Poovorawan Y: Entire genome characterization of human papillomavirus type 16 (HPV16) from infected Thai women with different cytological findings. Virus Genes 2009;39:30-38. 
-18 Combita AL, Touzé A, Bousarghin L, Christensen ND, Coursaget P: Identification of two cross-neutralizing linear epitopes within the L1 major capsid protein of human papillomaviruses. J Virol 2002;76:6480-6486.

-19 López-Saavedra A, González-Maya L, Poncede-León S, García-Carrancá A, Mohar A, Lizano M: Functional implication of sequence variation in the long control region and E2 gene among human papillomavirus type 18 variants. Arch Virol 2009;154:747-754.
20 Arias-Pulido H, Peyton CL, Torrez-Martínez N, Anderson DN, Wheeler CM: Human papillomavirus types 18 variants lineages in United States populations characterized by sequence analysis of LCRE6, E2 and L1 regions. Virology 2005;338: 22-34.

-21 Lizano M, De la Cruz-Hernandez E, Carrillo-Garcia A, Garcia-Carranca A, Ponce-deLeon-Rosales S, Duenas-Gonzalez A, Hernandez-HernadezDM,MoharA:Distribution of HPV16 and 18 intratypic variants in normal cytology, intraepithelial lesions, and cervical cancer in a Mexican population. Gynecol Oncol 2006;102:230-235.

$\checkmark 22$ Michels KB, zur Hausen H: HPV vaccine for all. Lancet 2009;374:268-270.
23 Alphs HH, Gambhira R, Karanam B, Roberts JN, Jagu S, Schiller JT, Zeng W, Jackson DC, Roden RBS: Protection against heterologous human papillomavirus challenge by a synthetic lipopeptide vaccine containing a broadly cross-neutralizing epitope of L2. PNAS 2008; 105:5850-5855.

24 Burk RD, Terai M, Gravitt PE, Brinton LA, Kurman RJ, Barnes WA, Greenberg MD, Hadjimichael OC, Fu L, McGowan L, Mortel R, Schwartz PE, Hildesheim A: Distribution of human papillomavirus types 16 and 18 variants in squamous cell carcinomas and adenocarcinomas of the cervix. Cancer Res 2003;63:7215-7220. 